\title{
Alignment-Based Compositional Semantics for Instruction Following
}

\author{
Jacob Andreas and Dan Klein \\ Computer Science Division \\ University of California, Berkeley \\ $\{j d a, k l e i n\}$ es.berkeley.edu
}

\begin{abstract}
This paper describes an alignment-based model for interpreting natural language instructions in context. We approach instruction following as a search over plans, scoring sequences of actions conditioned on structured observations of text and the environment. By explicitly modeling both the low-level compositional structure of individual actions and the high-level structure of full plans, we are able to learn both grounded representations of sentence meaning and pragmatic constraints on interpretation. To demonstrate the model's flexibility, we apply it to a diverse set of benchmark tasks. On every task, we outperform strong task-specific baselines, and achieve several new state-of-the-art results.
\end{abstract}

\section{Introduction}

In instruction-following tasks, an agent executes a sequence of actions in a real or simulated environment, in response to a sequence of natural language commands. Examples include giving navigational directions to robots and providing hints to automated game-playing agents. Plans specified with natural language exhibit compositionality both at the level of individual actions and at the overall sequence level. This paper describes a framework for learning to follow instructions by leveraging structure at both levels.

Our primary contribution is a new, alignmentbased approach to grounded compositional semantics. Building on related logical approaches (Reddy et al., 2014; Pourdamghani et al., 2014), we recast instruction following as a pair of nested, structured alignment problems. Given instructions and a candidate plan, the model infers a sequenceto-sequence alignment between sentences and atomic actions. Within each sentence-action pair, the model infers a structure-to-structure alignment between the syntax of the sentence and a graphbased representation of the action.

At a high level, our agent is a block-structured, graph-valued conditional random field, with alignment potentials to relate instructions to actions and transition potentials to encode the environment model (Figure 3). Explicitly modeling sequenceto-sequence alignments between text and actions allows flexible reasoning about action sequences, enabling the agent to determine which actions are specified (perhaps redundantly) by text, and which actions must be performed automatically (in order to satisfy pragmatic constraints on interpretation). Treating instruction following as a sequence prediction problem, rather than a series of independent decisions (Branavan et al., 2009; Artzi and Zettlemoyer, 2013), makes it possible to use general-purpose planning machinery, greatly increasing inferential power.

The fragment of semantics necessary to complete most instruction-following tasks is essentially predicate-argument structure, with limited influence from quantification and scoping. Thus the problem of sentence interpretation can reasonably be modeled as one of finding an alignment between language and the environment it describes. We allow this structure-to-structure alignmentan "overlay" of language onto the world-to be mediated by linguistic structure (in the form of dependency parses) and structured perception (in what we term grounding graphs). Our model thereby reasons directly about the relationship between language and observations of the environment, without the need for an intermediate logical representation of sentence meaning. This, in turn, makes it possible to incorporate flexible feature representations that have been difficult to integrate with previous work in semantic parsing.

We apply our approach to three established 


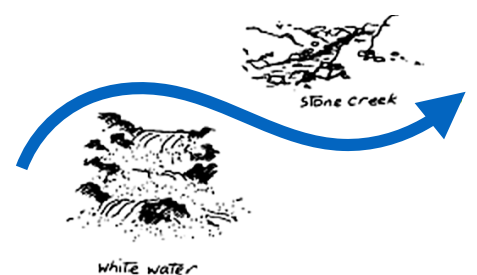

... right round the white water but stay quite close 'cause you don't otherwise you're going to be in that stone creek...

(a) Map reading

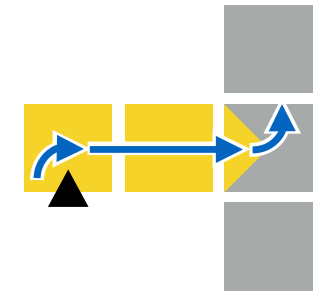

Go down the yellow hall. Turn left at the intersection of the yellow and the gray.

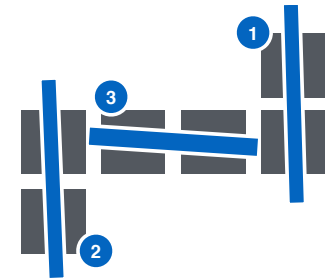

Clear the right column. Then the other column. Then the row.

(c) Puzzle solving

Figure 1: Example tasks handled by our framework. The tasks feature noisy text, over- and under-specification of plans, and challenging search problems.

instruction-following benchmarks: the map reading task of Vogel and Jurafsky (2010), the maze navigation task of MacMahon et al. (2006), and the puzzle solving task of Branavan et al. (2009). An example from each is shown in Figure 1. These benchmarks exhibit a range of qualitative properties-both in the length and complexity of their plans, and in the quantity and quality of accompanying language. Each task has been studied in isolation, but we are unaware of any published approaches capable of robustly handling all three. Our general model outperforms strong, task-specific baselines in each case, achieving relative error reductions of $15-20 \%$ over several state-of-the-art results. Experiments demonstrate the importance of our contributions in both compositional semantics and search over plans. We have released all code for this project at github.com/jacobandreas/instructions.

\section{Related work}

Existing work on instruction following can be roughly divided into two families: semantic parsers and linear policy estimators.

Semantic parsers Parser-based approaches (Chen and Mooney, 2011; Artzi and Zettlemoyer, 2013; Kim and Mooney, 2013) map from text into a formal language representing commands. These take familiar structured prediction models for semantic parsing (Zettlemoyer and Collins, 2005; Wong and Mooney, 2006), and train them with task-provided supervision. Instead of attempting to match the structure of a manually-annotated semantic parse, semantic parsers for instruction following are trained to maximize a reward signal provided by black-box execution of the predicted command in the environment. (It is possible to think of response-based learning for question answering (Liang et al., 2013) as a special case.)

This approach uses a well-studied mechanism for compositional interpretation of language, but is subject to certain limitations. Because the environment is manipulated only through black-box execution of the completed semantic parse, there is no way to incorporate current or future environment state into the scoring function. It is also in general necessary to hand-engineer a task-specific formal language for describing agent behavior. Thus it is extremely difficult to work with environments that cannot be modeled with a fixed inventory of predicates (e.g. those involving novel strings or arbitrary real quantities).

Much of contemporary work in this family is evaluated on the maze navigation task introduced by MacMahon et al. (2006). Dukes (2013) also introduced a "blocks world" task for situated parsing of spatial robot commands.

Linear policy estimators An alternative family of approaches is based on learning a policy over primitive actions directly (Branavan et al., 2009; Vogel and Jurafsky, 2010). ${ }^{1}$ Policybased approaches instantiate a Markov decision process representing the action domain, and apply standard supervised or reinforcement-learning approaches to learn a function for greedily selecting among actions. In linear policy approximators, natural language instructions are incorporated directly into state observations, and reading order

\footnotetext{
${ }^{1}$ This is distinct from semantic parsers in which greedy inference happens to have an interpretation as a policy (Vlachos and Clark, 2014).
} 
becomes part of the action selection process.

Almost all existing policy-learning approaches make use of an unstructured parameterization, with a single (flat) feature vector representing all text and observations. Such approaches are thus restricted to problems that are simple enough (and have small enough action spaces) to be effectively characterized in this fashion. While there is a great deal of flexibility in the choice of feature function (which is free to inspect the current and future state of the environment, the whole instruction sequence, etc.), standard linear policy estimators have no way to model compositionality in language or actions.

Agents in this family have been evaluated on a variety of tasks, including map reading (Anderson et al., 1991) and gameplay (Branavan et al., 2009).

Though both families address the same class of instruction-following problems, they have been applied to a totally disjoint set of tasks. It should be emphasized that there is nothing inherent to policy learning that prevents the use of compositional structure, and nothing inherent to general compositional models that prevents more complicated dependence on environment state. Indeed, previous work (Branavan et al., 2011; Narasimhan et al., 2015) uses aspects of both to solve a different class of gameplay problems. In some sense, our goal in this paper is simply to combine the strengths of semantic parsers and linear policy estimators for fully general instruction following. As we shall see, however, this requires changes to many aspects of representation, learning and inference.

\section{Representations}

We wish to train a model capable of following commands in a simulated environment. We do so by presenting the model with a sequence of training pairs $(\mathbf{x}, \mathbf{y})$, where each $\mathbf{x}$ is a sequence of natural language instructions $\left(x_{1}, x_{2}, \ldots, x_{m}\right)$, e.g.:

(Go down the yellow hall., Turn left., ...)

and each $\mathbf{y}$ is a demonstrated action sequence $\left(y_{1}, y_{2}, \ldots, y_{n}\right)$, e.g.:

$$
\text { (rotate }(90), \text { move }(2), \ldots)
$$

Given a start state, $\mathbf{y}$ can equivalently be characterized by a sequence of (state, action, state) (a) Text

\section{Go down the yellow hall}

(b) Syntax

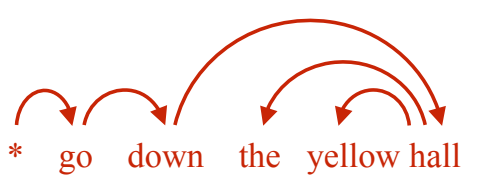

(c) Alignment

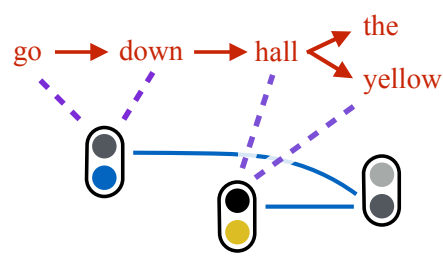

(d) Perception

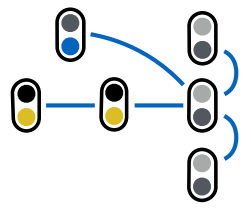

move (2)

(e) Environment

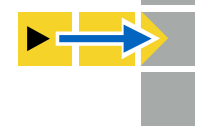

Figure 2: Structure-to-structure alignment connecting a single sentence (via its syntactic analysis) to the environment state (via its grounding graph). The connecting alignments take the place of a traditional semantic parse and allow flexible, feature-driven linking between lexical primitives and perceptual factors.

triples resulting from execution of the environment model. An example instruction is shown in Figure 2a. An example action, situated in the environment where it occurs, is shown in Figure 2e.

Our model performs compositional interpretation of instructions by leveraging existing structure inherent in both text and actions. Thus we interpret $x_{i}$ and $y_{j}$ not as raw strings and primitive actions, but rather as structured objects.

Linguistic structure We assume access to a pretrained parser, and in particular that each of the instructions $x_{i}$ is represented by a tree-structured dependency parse. An example is shown in Figure $2 b$.

Action structure By analogy to the representation of instructions as parse trees, we assume that each (state, action, state) triple (provided by the environment model) can be characterized by a grounding graph. The structure and content of this representation is task-specific. An example grounding graph for the maze navigation task is 
shown in Figure $2 \mathrm{~d}$. The example contains a node corresponding to the primitive action move (2) (in the upper left), and several nodes corresponding to locations in the environment that are visible after the action is performed.

Each node in the graph (and, though not depicted, each edge) is decorated with a list of features. These features might be simple indicators (e.g. whether the primitive action performed was move or rotate), real values (the distance traveled) or even string-valued (English-language names of visible landmarks, if available in the environment description). Formally, a grounding graph consists of a tuple $\left(V, E, \mathcal{L}, f_{V}, f_{E}\right)$, with

- $V$ a set of vertices

- $E \in V \times V$ a set of (directed) edges

- $\mathcal{L}$ a space of labels (numbers, strings, etc.)

- $f_{V}: V \rightarrow 2^{\mathcal{L}}$ a vertex feature function

$-f_{E}: E \rightarrow 2^{\mathcal{L}}$ an edge feature function

In this paper we have tried to remain agnostic to details of graph construction. Our goal with the grounding graph framework is simply to accommodate a wider range of modeling decisions than allowed by existing formalisms. Graphs might be constructed directly, given access to a structured virtual environment (as in all experiments in this paper), or alternatively from outputs of a perceptual system. For our experiments, we have remained as close as possible to task representations described in the existing literature. Details for each task can be found in the accompanying software package.

Graph-based representations are extremely common in formal semantics (Jones et al., 2012; Reddy et al., 2014), and the version presented here corresponds to a simple generalization of familiar formal methods. Indeed, if $\mathcal{L}$ is the set of all atomic entities and relations, $f_{V}$ returns a unique label for every $v \in V$, and $f_{E}$ always returns a vector with one active feature, we recover the existentially-quantified portion of first order logic exactly, and in this form can implement large parts of classical neo-Davidsonian semantics (Parsons, 1990) using grounding graphs.

Crucially, with an appropriate choice of $\mathcal{L}$ this formalism also makes it possible to go beyond settheoretic relations, and incorporate string-valued features (like names of entities and landmarks) and real-valued features (like colors and positions) as well.

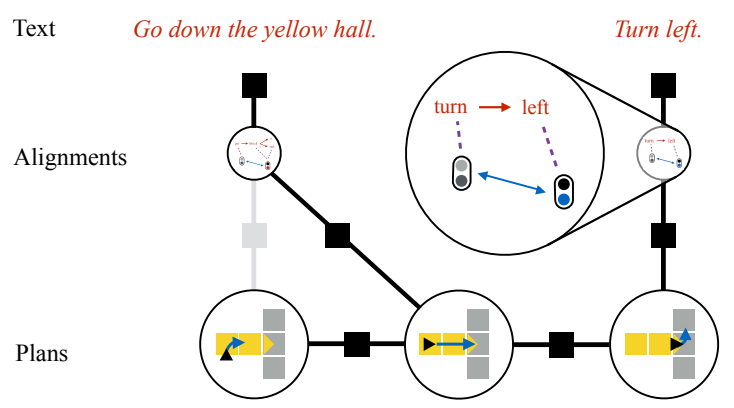

Figure 3: Our model is a conditional random field that describes distributions over state-action sequences conditioned on input text. Each variable's domain is a structured value. Sentences align to a subset of the state-action sequences, with the rest of the states filled in by pragmatic (planning) implication. State-to-state structure represents planning constraints (environment model) while state-to-text structure represents compositional alignment. All potentials are log-linear and feature-driven.

Lexical semantics We must eventually combine features provided by parse trees with features provided by the environment. Examples here might include simple conjunctions (word=yellow $\wedge$ $r g b=(0.5,0.5,0.0))$ or more complicated computations like edit distance between landmark names and lexical items. Features of the latter kind make it possible to behave correctly in environments containing novel strings or other features unseen during training.

This aspect of the syntax-semantics interface has been troublesome for some logic-based approaches: while past work has used related machinery for selecting lexicon entries (Berant and Liang, 2014) or for rewriting logical forms (Kwiatkowski et al., 2013), the relationship between text and the environment has ultimately been mediated by a discrete (and indeed finite) inventory of predicates. Several recent papers have investigated simple grounded models with realvalued output spaces (Andreas and Klein, 2014; McMahan and Stone, 2015), but we are unaware of any fully compositional system in recent literature that can incorporate observations of these kinds.

Formally, we assume access to a joining feature function $\phi:\left(2^{\mathcal{L}} \times 2^{\mathcal{L}}\right) \rightarrow \mathbb{R}^{d}$. As with grounding graphs, our goal is to make the general framework as flexible as possible, and for individual experiments have chosen $\phi$ to emulate modeling decisions from previous work. 


\section{Model}

As noted in the introduction, we approach instruction following as a sequence prediction problem. Thus we must place a distribution over sequences of actions conditioned on instructions. We decompose the problem into two components, describing interlocking models of "path structure" and "action structure". Path structure captures how sequences of instructions give rise to sequences of actions, while action structure captures the compositional relationship between individual utterances and the actions they specify.

\section{Path structure: aligning utterances to actions}

The high-level path structure in the model is depicted in Figure 3. Our goal here is to permit both under- and over-specification of plans, and to expose a planning framework which allows plans to be computed with lookahead (i.e. non-greedily).

These goals are achieved by introducing a sequence of latent alignments between instructions and actions. Consider the multi-step example in Figure 1b. If the first instruction go down the yellow hall were interpreted immediately, we would have a presupposition failure - the agent is facing a wall, and cannot move forward at all. Thus an implicit rot ate action, unspecified by text, must be performed before any explicit instructions can be followed.

To model this, we take the probability of a (text, plan, alignment) triple to be log-proportional to the sum of two quantities:

1. a path-only score $\psi(n ; \theta)+\sum_{j} \psi\left(y_{j} ; \theta\right)$

2. a path-and-text score, itself the sum of all pair scores $\psi\left(x_{i}, y_{j} ; \theta\right)$ licensed by the alignment

(1) captures our desire for pragmatic constraints on interpretation, and provides a means of encoding the inherent plausibility of paths. We take $\psi(n ; \theta)$ and $\psi(y ; \theta)$ to be linear functions of $\theta$. (2) provides context-dependent interpretation of text by means of the structured scoring function $\psi(x, y ; \theta)$, described in the next section.

Formally, we associate with each instruction $x_{i}$ a sequence-to-sequence alignment variable $a_{i} \in$ $1 \ldots n$ (recalling that $n$ is the number of actions).
Then we have ${ }^{2}$

$$
\begin{aligned}
& p(\mathbf{y}, \mathbf{a} \mid \mathbf{x} ; \theta) \propto \exp \left\{\psi(n)+\sum_{j=1}^{n} \psi\left(y_{j}\right)\right. \\
&\left.+\sum_{i=1}^{m} \sum_{j=1}^{n} \mathbf{1}\left[a_{j}=i\right] \psi\left(x_{i}, y_{j}\right)\right\}
\end{aligned}
$$

We additionally place a monotonicity constraint on the alignment variables. This model is globally normalized, and for a fixed alignment is equivalent to a linear-chain CRF. In this sense it is analogous to IBM Model I (Brown et al., 1993), with the structured potentials $\psi\left(x_{i}, y_{j}\right)$ taking the place of lexical translation probabilities. While alignment models from machine translation have previously been used to align words to fragments of semantic parses (Wong and Mooney, 2006; Pourdamghani et al., 2014), we are unaware of such models being used to align entire instruction sequences to demonstrations.

\section{Action structure: aligning words to percepts}

Intuitively, this scoring function $\psi(x, y)$ should capture how well a given utterance describes an action. If neither the utterances nor the actions had structure (i.e. both could be represented with simple bags of features), we would recover something analogous to the conventional policy-learning approach. As structure is essential for some of our tasks, $\psi(x, y)$ must instead fill the role of a semantic parser in a conventional compositional model.

Our choice of $\psi(x, y)$ is driven by the following fundamental assumptions: Syntactic relations approximately represent semantic relations. Syntactic proximity implies relational proximity. In this view, there is an additional hidden structure-tostructure alignment between the grounding graph and the parsed text describing it. ${ }^{3}$ Words line up with nodes, and dependencies line up with relations. Visualizations are shown in Figure $2 c$ and the zoomed-in portion of Figure 3.

As with the top-level alignment variables, this approach can viewed as a simple relaxation of a familiar model. CCG-based parsers assume that syntactic type strictly determines semantic type,

\footnotetext{
${ }^{2}$ Here and the remainder of this paper, we suppress the dependence of the various potentials on $\theta$ in the interest of readability.

${ }^{3}$ It is formally possible to regard the sequence-tosequence and structure-to-structure alignments as a single (structured) random variable. However, the two kinds of alignments are treated differently for purposes of inference, so it is useful to maintain a notational distinction.
} 
and that each lexical item is associated with a small set of functional forms. Here we simply allow all words to license all predicates, multiple words to specify the same predicate, and some edges to be skipped. We instead rely on a scoring function to impose soft versions of the hard constraints typically provided by a grammar. Related models have previously been used for question answering (Reddy et al., 2014; Pasupat and Liang, 2015).

For the moment let us introduce variables $b$ to denote these structure-to-structure alignments. (As will be seen in the following section, it is straightforward to marginalize over all choices of $b$. Thus the structure-to-structure alignments are never explicitly instantiated during inference, and do not appear in the final form of $\psi(x, y)$.) For a fixed alignment, we define $\psi(x, y, b)$ according to a recurrence relation. Let $x^{i}$ be the $i$ th word of the sentence, and let $y^{j}$ be the $j$ th node in the action graph (under some topological ordering). Let $c(i)$ and $c(j)$ give the indices of the dependents of $x^{i}$ and children of $y^{j}$ respectively. Finally, let $x^{i k}$ and $y^{j l}$ denote the associated dependency type or relation. Define a "descendant" function:

$$
d(i, j)=\{(k, l): k \in c(i), l \in c(j),(k, l) \in b\}
$$

Then,

$$
\begin{aligned}
& \psi\left(x^{i}, y^{j}, b\right)=\exp \left\{\theta^{\top} \phi\left(x^{i}, y^{j}\right)\right. \\
& \left.+\sum_{(k, l) \in d(x, y)}\left[\theta^{\top} \phi\left(x^{i k}, y^{j l}\right) \cdot \psi\left(x^{k}, y^{l}, b\right)\right]\right\}
\end{aligned}
$$

This is just an unnormalized synchronous derivation between $x$ and $y$ - at any aligned (node, word) pair, the score for the entire derivation is the score produced by combining that word and node, times the scores at all the aligned descendants. Observe that as long as there are no cycles in the dependency parse, it is perfectly acceptable for the relation graph to contain cycles and even self-loopsthe recurrence still bottoms out appropriately.

\section{Learning and inference}

Given a sequence of training pairs $(\mathbf{x}, \mathbf{y})$, we wish to find a parameter setting that maximizes $p(\mathbf{y} \mid \mathbf{x} ; \theta)$. If there were no latent alignments $a$ or $b$, this would simply involve minimization of a convex objective. The presence of latent variables complicates things. Ideally, we would like

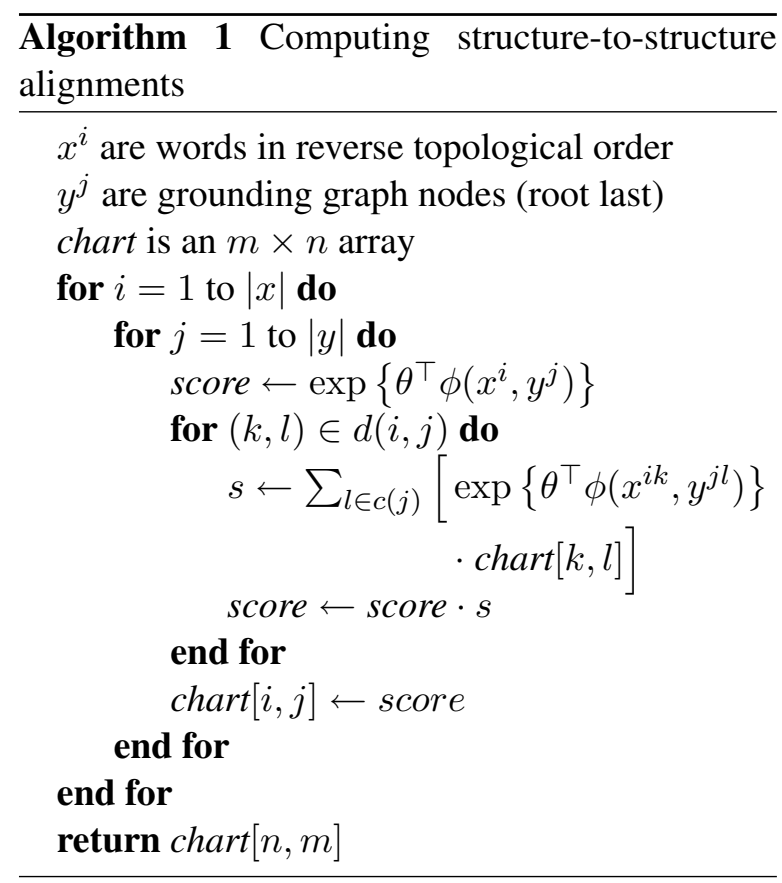

to sum over the latent variables, but that sum is intractable. Instead we make a series of variational approximations: first we replace the sum with a maximization, then perform iterated conditional modes, alternating between maximization of the conditional probability of $\mathbf{a}$ and $\theta$. We begin by initializing $\theta$ randomly.

As noted in the preceding section, the variable $b$ does not appear in these equations. Conditioned on $\mathbf{a}$, the sum over structure-to-structure $\psi(x, y)=\sum_{b} \psi(x, y, b)$ can be performed exactly using a simple dynamic program which runs in time $\mathcal{O}(|x||y|)$ (assuming out-degree bounded by a constant, and with $|x|$ and $|y|$ the number of words and graph nodes respectively). This is Algorithm 1 .

In our experiments, $\theta$ is optimized using LBFGS (Liu and Nocedal, 1989). Calculation of the gradient with respect to $\theta$ requires computation of a normalizing constant involving the sum over $p\left(\mathbf{x}, \mathbf{y}^{\prime}, \mathbf{a}\right)$ for all $\mathbf{y}^{\prime}$. While in principle the normalizing constant can be computed using the forward algorithm, in practice the state spaces under consideration are so large that even this is intractable. Thus we make an additional approximation, constructing a set $\tilde{Y}$ of alternative actions and taking

$$
p(\mathbf{y}, \mathbf{a} \mid \mathbf{x}) \approx \sum_{j=1}^{n} \frac{\exp \left\{\psi\left(y_{j}\right)+\sum_{i=1}^{m} \mathbf{1}\left[a_{i}=j\right] \psi\left(x_{i}, y_{i}\right)\right\}}{\sum_{\tilde{y} \in \tilde{Y}} \exp \left\{\psi(\tilde{y})+\sum_{i=1}^{m} \mathbf{1}\left[a_{i}=j\right] \psi\left(x_{i}, \tilde{y}\right)\right\}}
$$


$\tilde{Y}$ is constructed by sampling alternative actions from the environment model. Meanwhile, maximization of a can be performed exactly using the Viterbi algorithm, without computation of normalizers.

Inference at test time involves a slightly different pair of optimization problems. We again perform iterated conditional modes, here on the alignments a and the unknown output path $\mathbf{y}$. Maximization of $\mathbf{a}$ is accomplished with the Viterbi algorithm, exactly as before; maximization of $\mathbf{y}$ also uses the Viterbi algorithm, or a beam search when this is computationally infeasible. If bounds on path length are known, it is straightforward to adapt these dynamic programs to efficiently consider paths of all lengths.

\section{Evaluation}

As one of the main advantages of this approach is its generality, we evaluate on several different benchmark tasks for instruction following. These exhibit great diversity in both environment structure and language use. We compare our full system to recent state-of-the-art approaches to each task. In the introduction, we highlighted two core aspects of our approach to semantics: compositionality (by way of grounding graphs and structure-to-structure alignments) and planning (by way of inference with lookahead and sequence-to-sequence alignments). To evaluate these, we additionally present a pair of ablation experiments: no grounding graphs (an agent with an unstructured representation of environment state), and no planning (a reflex agent with no lookahead).

Map reading Our first application is the map navigation task established by Vogel and Jurafsky (2010), based on data collected for a psychological experiment by Anderson et al. (1991) (Figure 1a). Each training datum consists of a map with a designated starting position, and a collection of landmarks, each labeled with a spatial coordinate and a string name. Names are not always unique, and landmarks in the test set are never observed during training. This map is accompanied by a set of instructions specifying a path from the starting position to some (unlabeled) destination point. These instruction sets are informal and redundant, involving as many as a hundred utterances. They are transcribed from spoken text, so grammatical errors, disfluencies, etc. are common. This is a

\begin{tabular}{lccc}
\hline & $\mathrm{P}$ & $\mathrm{R}$ & $\mathrm{F}_{1}$ \\
\hline Vogel and Jurafsky (2010) & 0.46 & 0.51 & $\mathbf{0 . 4 8}$ \\
Andreas and Klein (2014) & 0.43 & 0.51 & 0.45 \\
---- & $-\overline{4}$ & $-\overline{0}-\overline{-}-$ & $--\overline{-}-$ \\
Model [no planning] & 0.44 & 0.46 & 0.45 \\
Model [no grounding graphs] & 0.52 & 0.52 & 0.52 \\
Model [full] & 0.51 & 0.60 & $\mathbf{0 . 5 5}$ \\
\hline
\end{tabular}

Table 1: Evaluation results for the map-reading task. $\mathrm{P}$ is precision, $R$ is recall and $F_{1}$ is F-measure. Scores are calculated with respect to transitions between landmarks appearing in the reference path (for details see Vogel and Jurafsky (2010)). We use the same train / test split. Some variant of our model achieves the best published results on all three metrics.

\begin{tabular}{lc}
\hline Feature & Weight \\
\hline word=top $\wedge$ side=North & 1.31 \\
word=top $\wedge$ side=South & 0.61 \\
word=top $\wedge$ side=East & -0.93 \\
------------- & $-\overline{-}--$ \\
dist $=0$ & 4.51 \\
dist $=1$ & 2.78 \\
dist $=4$ & 1.54 \\
\hline
\end{tabular}

Table 2: Learned feature values. The model learns that the word top often instructs the navigator to position itself above a landmark, occasionally to position itself below a landmark, but rarely to the side. The bottom portion of the table shows learned text-independent constraints: given a choice, near destinations are preferred to far ones (so shorter paths are preferred overall).

prime example of a domain that does not lend itself to logical representation-grammars may be too rigid, and previously-unseen landmarks and real-valued positions are handled more easily with feature machinery than predicate logic.

The map task was previously studied by Vogel and Jurafsky (2010), who implemented SARSA with a simple set of features. By combining these features with our alignment model and search procedure, we achieve state-of-the-art results on this task by a substantial margin (Table 1).

Some learned feature values are shown in Table 2. The model correctly infers cardinal directions (the example shows the preferred side of a destination landmark modified by the word top). Like Vogel et al., we see support for both allocentric references (you are on top of the hill) and egocentric references (the hill is on top of you). We can also see pragmatics at work: the model learns useful text-independent constraints-in this case, that near destinations should be preferred to far ones.

Maze navigation The next application we consider is the maze navigation task of MacMahon et al. (2006) (Figure 1b). Here, a virtual agent is sit- 


\begin{tabular}{|c|c|}
\hline & Success $(\%)$ \\
\hline Kim and Mooney (2012) & 57.2 \\
\hline Chen (2012) & 57.3 \\
\hline$\overline{\text { Model [no planning] }}$ & $-\overline{58.9}--$ \\
\hline Model [no grounding graphs] & 51.7 \\
\hline Model [full] & 59.6 \\
\hline Kim and Mooney (2013) [reranked] & 62.8 \\
\hline Artzi et al. (2014) [semi-supervised] & 65.3 \\
\hline
\end{tabular}

Table 3: Evaluation results for the maze navigation task. "Success" shows the percentage of actions resulting in a correct position and orientation after observing a single instruction. We use the leave-one-map-out evaluation employed by previous work. ${ }^{4}$ All systems are trained on full action sequences. Our model outperforms several task-specific baselines, as well as a baseline with path structure but no action structure.

uated in a maze (whose hallways are distinguished with various wallpapers, carpets, and the presence of a small set of standard objects), and again given instructions for getting from one point to another. This task has been the subject of focused attention in semantic parsing for several years, resulting in a variety of sophisticated approaches.

Despite superficial similarity to the previous navigation task, the language and plans required for this task are quite different. The proportion of instructions to actions is much higher (so redundancy much lower), and the interpretation of language is highly compositional.

As can be seen in Table 3, we outperform a number of systems purpose-built for this navigation task. We also outperform both variants of our system, most conspicuously the variant without grounding graphs. This highlights the importance of compositional structure. Recent work by Kim and Mooney (2013) and Artzi et al. (2014) has achieved better results; these systems make use of techniques and resources (respectively, discriminative reranking and a seed lexicon of handannotated logical forms) that are largely orthogonal to the ones used here, and might be applied to improve our own results as well.

Puzzle solving The last task we consider is the Crossblock task studied by Branavan et al. (2009) (Figure 1c). Here, again, natural language is used to specify a sequence of actions, in this case the solution to a simple game. The environment is simple enough to be captured with a flat feature

\footnotetext{
${ }^{4}$ We specifically targeted the single-sentence version of this evaluation, as an alternative full-sequence evaluation does not align precisely with our data condition.
}

\begin{tabular}{lcc}
\hline & Match (\%) & Success (\%) \\
\hline No text & 54 & 78 \\
Branavan '09 & $\mathbf{6 3}$ & - \\
$--\overline{-}------$ & ---- \\
Model [no planning] & 64 & 66 \\
Model [full] & $\mathbf{7 0}$ & $\mathbf{8 6}$ \\
\hline
\end{tabular}

Table 4: Results for the puzzle solving task. "Match" shows the percentage of predicted action sequences that exactly match the annotation. "Success" shows the percentage of predicted action sequences that result in a winning game configuration, regardless of the action sequence performed. Following Branavan et al. (2009), we average across five random train / test folds. Our model achieves state-of-the-art results on this task.

representation, so there is no distinction between the full model and the variant without grounding graphs.

Unlike the other tasks we consider, Crossblock is distinguished by a challenging associated search problem. Here it is nontrivial to find any sequence that eliminates all the blocks (the goal of the puzzle). Thus this example allows us measure the effectiveness of our search procedure.

Results are shown in Table 4. As can be seen, our model achieves state-of-the-art performance on this task when attempting to match the humanspecified plan exactly. If we are purely concerned with task completion (i.e. solving the puzzle, perhaps not with the exact set of moves specified in the instructions) we can measure this directly. Here, too, we substantially outperform a no-text baseline. Thus it can be seen that text induces a useful heuristic, allowing the model to solve a considerable fraction of problem instances not solved by naïve beam search.

The problem of inducing planning heuristics from side information like text is an important one in its own right, and future work might focus specifically on coupling our system with a more sophisticated planner. Even at present, the results in this section demonstrate the importance of lookahead and high-level reasoning in instruction following.

\section{Conclusion}

We have described a new alignment-based compositional model for following sequences of natural language instructions, and demonstrated the effectiveness of this model on a variety of tasks. A fully general solution to the problem of contextual interpretation must address a wide range of wellstudied problems, but the work we have described 
here provides modular interfaces for the study of a number of fundamental linguistic issues from a machine learning perspective. These include:

Pragmatics How do we respond to presupposition failures, and choose among possible interpretations of an instruction disambiguated only by context? The mechanism provided by the sequence-prediction architecture we have described provides a simple answer to this question, and our experimental results demonstrate that the learned pragmatics aid interpretation of instructions in a number of concrete ways: ambiguous references are resolved by proximity in the map reading task, missing steps are inferred from an environment model in the maze navigation task, and vague hints are turned into real plans by knowledge of the rules in Crossblock. A more comprehensive solution might explicitly describe the process by which instruction-givers' own beliefs (expressed as distributions over sequences) give rise to instructions.

Compositional semantics The graph alignment model of semantics presented here is an expressive and computationally efficient generalization of classical logical techniques to accommodate environments like the map task, or those explored in our previous work (Andreas and Klein, 2014). More broadly, our model provides a compositional approach to semantics that does not require an explicit formal language for encoding sentence meaning. Future work might extend this approach to tasks like question answering, where logicbased approaches have been successful.

Our primary goal in this paper has been to explore methods for integrating compositional semantics and the pragmatic context provided by sequential structures. While there is a great deal of work left to do, we find it encouraging that this general approach results in substantial gains across multiple tasks and contexts.

\section{Acknowledgments}

The authors would like to thank S.R.K. Branavan for assistance with the Crossblock evaluation. The first author is supported by a National Science Foundation Graduate Fellowship.

\section{References}

Anne H. Anderson, Miles Bader, Ellen Gurman Bard, Elizabeth Boyle, Gwyneth Doherty, Simon Garrod, Stephen Isard, Jacqueline Kowtko, Jan McAllister, Jim Miller, et al. 1991. The HCRC map task corpus. Language and speech, 34(4):351-366.

Jacob Andreas and Dan Klein. 2014. Grounding language with points and paths in continuous spaces. In Proceedings of the Conference on Natural Language Learning.

Yoav Artzi and Luke Zettlemoyer. 2013. Weakly supervised learning of semantic parsers for mapping instructions to actions. Transactions of the Association for Computational Linguistics, 1(1):49-62.

Yoav Artzi, Dipanjan Das, and Slav Petrov. 2014. Learning compact lexicons for CCG semantic parsing. In Proceedings of the Conference on Empirical Methods in Natural Language Processing, pages 1273-1283, Doha, Qatar, October. Association for Computational Linguistics.

Jonathan Berant and Percy Liang. 2014. Semantic parsing via paraphrasing. In Proceedings of the Annual Meeting of the Association for Computational Linguistics, page 92.

S.R.K. Branavan, Harr Chen, Luke S. Zettlemoyer, and Regina Barzilay. 2009. Reinforcement learning for mapping instructions to actions. In Proceedings of the Annual Meeting of the Association for Computational Linguistics, pages 82-90. Association for Computational Linguistics.

S.R.K. Branavan, David Silver, and Regina Barzilay. 2011. Learning to win by reading manuals in a Monte-Carlo framework. In Proceedings of the $\mathrm{Hu}$ man Language Technology Conference of the Association for Computational Linguistics, pages 268277.

Peter Brown, Vincent Della Pietra, Stephen Della Pietra, and Robert Mercer. 1993. The mathematics of statistical machine translation: Parameter estimation. Computational Linguistics, 19(2):263-311, June.

David L. Chen and Raymond J. Mooney. 2011. Learning to interpret natural language navigation instructions from observations. In Proceedings of the Meeting of the Association for the Advancement of Artificial Intelligence, volume 2, pages 1-2.

David L Chen. 2012. Fast online lexicon learning for grounded language acquisition. In Proceedings of the Annual Meeting of the Association for Computational Linguistics, pages 430-439.

Kais Dukes. 2013. Semantic annotation of robotic spatial commands. In Language and Technology Conference (LTC). 
Bevan Jones, Jacob Andreas, Daniel Bauer, Karl Moritz Hermann, and Kevin Knight. 2012. Semantics-based machine translation with hyperedge replacement grammars. In Proceedings of the International Conference on Computational Linguistics, pages 1359-1376.

Joohyun Kim and Raymond J. Mooney. 2012. Unsupervised PCFG induction for grounded language learning with highly ambiguous supervision. In Proceedings of the Conference on Empirical Methods in Natural Language Processing, pages 433-444.

Joohyun Kim and Raymond J. Mooney. 2013. Adapting discriminative reranking to grounded language learning. In Proceedings of the Annual Meeting of the Association for Computational Linguistics.

Tom Kwiatkowski, Eunsol Choi, Yoav Artzi, and Luke Zettlemoyer. 2013. Scaling semantic parsers with on-the-fly ontology matching. In Proceedings of the Conference on Empirical Methods in Natural Language Processing.

Percy Liang, Michael I. Jordan, and Dan Klein. 2013. Learning dependency-based compositional semantics. Computational Linguistics, 39(2):389-446.

Dong Liu and Jorge Nocedal. 1989. On the limited memory BFGS method for large scale optimization. Mathematical Programming, 45(1-3):503-528.

Matt MacMahon, Brian Stankiewicz, and Benjamin Kuipers. 2006. Walk the talk: Connecting language, knowledge, and action in route instructions. Proceedings of the Meeting of the Association for the Advancement of Artificial Intelligence, 2(6):4.

Brian McMahan and Matthew Stone. 2015. A Bayesian model of grounded color semantics. Transactions of the Association for Computational Linguistics, 3:103-115.

Karthik Narasimhan, Tejas Kulkarni, and Regina Barzilay. 2015. Language understanding for textbased games using deep reinforcement learning. In Proceedings of the Conference on Empirical Methods in Natural Language Processing.

Terence Parsons. 1990. Events in the semantics of English. MIT Press.

Panupong Pasupat and Percy Liang. 2015. Compositional semantic parsing on semi-structured tables. In Proceedings of the Annual Meeting of the Association for Computational Linguistics.

Nima Pourdamghani, Yang Gao, Ulf Hermjakob, and Kevin Knight. 2014. Aligning english strings with abstract meaning representation graphs. In Proceedings of the Conference on Empirical Methods in Natural Language Processing.

Siva Reddy, Mirella Lapata, and Mark Steedman. 2014. Large-scale semantic parsing without question-answer pairs. Transactions of the Association for Computational Linguistics, 2:377-392.
Andreas Vlachos and Stephen Clark. 2014. A new corpus and imitation learning framework for contextdependent semantic parsing. Transactions of the Association for Computational Linguistics, 2:547-559.

Adam Vogel and Dan Jurafsky. 2010. Learning to follow navigational directions. In Proceedings of the Annual Meeting of the Association for Computational Linguistics, pages 806-814. Association for Computational Linguistics.

Yuk Wah Wong and Raymond Mooney. 2006. Learning for semantic parsing with statistical machine translation. In Proceedings of the Human Language Technology Conference of the North American Chapter of the Association for Computational Linguistics, pages 439-446, New York, New York.

Luke S. Zettlemoyer and Michael Collins. 2005. Learning to map sentences to logical form: Structured classification with probabilistic categorial grammars. In Proceedings of the Conference on Uncertainty in Artificial Intelligence, pages 658-666. 